\title{
Optical Control of the Magnetic Anisotropy of Ferromagnetic Bilayered Manganites
}

\author{
S. Tomimoto, ${ }^{1}$ M. Matsubara, ${ }^{1}$ T. Ogasawara, ${ }^{1}$ H. Okamoto, ${ }^{1,2}$ T. Kimura, ${ }^{3, *}$ and Y. Tokura ${ }^{1,3,4}$ \\ ${ }^{1}$ Correlated Electron Research Center (CERC), National Institute of Advanced Industrial Science and Technology (AIST), \\ Tsukuba 305-8562, Japan \\ ${ }^{2}$ Department of Advanced Materials Science, University of Tokyo, Kashiwa 277-8561, Japan \\ ${ }^{3}$ Department of Applied Physics, University of Tokyo, Tokyo 113-8656, Japan \\ ${ }^{4}$ Spin Superstructure Project (SSS), ERATO, Japan Science and Technology Corporation (JST), Tsukuba 305-0046, Japan
}

(Received 25 February 2006; published 5 January 2007)

\begin{abstract}
Optical manipulation of the magnetic anisotropy is demonstrated for bilayered manganites, $\mathrm{La}_{2-2 x} \mathrm{Sr}_{1+2 x} \mathrm{Mn}_{2} \mathrm{O}_{7}$, by means of femtosecond Kerr-rotation measurements. Upon the photoexcitation on the $x=0.32$ crystal, the magnetization exhibits the precessional motion for about $1 \mathrm{~ns}$, revealing the directional change of the magnetocrystalline anisotropy from the $c$ axis to the $a b$ plane. This change of the anisotropy induces the nonthermal decrease of the $c$-axis magnetization component for about $1 \mathrm{~ns}$.
\end{abstract}

Optical control of correlated spin systems in condensed matter such as ferromagnets and antiferromagnets has been one of the major issues in the field of spintronics. The studies to date have revealed that there are two types of spin controls. One is the control of the spin correlation, which can be observed in ferromagnets as changes in macroscopic magnetization. Both the optically induced magnetization [1] and demagnetization [2-6] phenomena have been observed so far. The other is the control of the magnetic anisotropy which governs the direction of the spin moments. Recently, ultrafast spin reorientation in an antiferromagnet with an intense laser pulse has been reported [7]. In ferromagnets, the anisotropy change manifests itself as the precessional motion of magnetization which is driven by the impulsive change of the anisotropy field $[6,8,9]$. This field is related to the shape anisotropy of the specimens [10], and its changes are derived from the thermal magnetization decrease induced with the photoirradiation.

In principle, the anisotropy field also comes from the magnetocrystalline anisotropy which is generally explained by the spin-orbit coupling or the anisotropic exchange interactions [11,12]. Thus, the change of the anisotropy can be expected if the electronic (especially orbital) state is modulated with the optical excitation. In this Letter, we report the observation of this new type of anisotropy change. It has been observed in a bilayered perovskite manganite, $\mathrm{La}_{2-2 x} \mathrm{Sr}_{1+2 x} \mathrm{Mn}_{2} \mathrm{O}_{7} \quad(x=0.32)$ which is located very close to the boundary of two ferromagnetic phases with different anisotropy. The photoinduced crossover of this phase boundary has been revealed in the temporal evolution of the magnetization.

Figure 1(a) shows the schematic phase diagram of $\mathrm{La}_{2-2 x} \mathrm{Sr}_{1+2 x} \mathrm{Mn}_{2} \mathrm{O}_{7}$ [13,14]. It exhibits ferromagnetic phases of Mn spins below $T_{c}$ around $100 \mathrm{~K}$. It is known that the favored direction of the spins sensitively depends on the hole doping level $x$. At $x=0.4$, the magnetization easy axis is parallel to the $a b$ plane (the bilayer of $\mathrm{MnO}_{6}$ octahedra), and large magnetic anisotropy appears between parallel and perpendicular to this plane [Fig. 1(b)]. This anisotropy is alleviated with decrease of $x$, and the easyaxis turns to the $c$ axis between $x=0.33$ and 0.32 [see Figs. 1(c) and 1(d)]. This change is known to be correlated with the orbital occupancy of an $e_{g}$ electron of $\mathrm{Mn}^{3+}\left(d^{4}\right)$ which changes from $d_{x^{2}-y^{2}}$ to $d_{3 z^{2}-r^{2}}$ as represented in Fig. 1(a) $[13,15]$. Consequently, we can anticipate the dynamic change of the anisotropy direction when the system is optically excited in the vicinity of the phase boundary, as a result of photodoping of holes into the $e_{g}$ band.

We have investigated the dynamics of magnetization after photoirradiation by a fs pump-probe method, and detected the temporal change of the polarization rotation $\theta_{K}$ due to the magneto-optical Kerr effect. The light source was a Ti:sapphire regenerative amplifier with the pulse width of $100 \mathrm{fs}$ and the repetition rate of $1 \mathrm{kHz}$. The fundamental output of this system $(1.55 \mathrm{eV})$ was used for the pump pulse, which is expected to generate electronhole pairs within the $e_{g}$ band [16]. For the probe, the second harmonic $(3.1 \mathrm{eV})$ was used. The samples are single crystals of $\mathrm{La}_{2-2 x} \mathrm{Sr}_{1+2 x} \mathrm{Mn}_{2} \mathrm{O}_{7}$ of the thickness around $1 \mathrm{~mm}$ which were grown by the floating-zone method. The optical measurement was done with the reflection light from cleaved $a b$ surfaces of the samples, and it enables us to monitor the $c$-axis component of the magnetization. A magnetic field is applied on the samples along the $c$ axis up to $3 \mathrm{~T}$ by a superconducting magnet. The time resolution of the pump-probe system is about $200 \mathrm{fs}$, and the angular resolution of the polarization rotation change $\Delta \theta_{K}$ is better than $0.1 \mathrm{mdeg}$. As shown in the $\theta_{K}$ spectra of Fig. 1(e), the $\theta_{K}$ is enhanced around the probe photon energy $(3.1 \mathrm{eV})$ owing to charge transfer transitions from $\mathrm{O} 2 p$ to $\mathrm{Mn} 3 d$ levels. It reflects the magnetization (the $c$-axis component) quite well, as assured from the comparison of the temperature dependence of the $\theta_{K}$ and the magnetization [Fig. 1(f)]. The penetration depth of the probe light is 


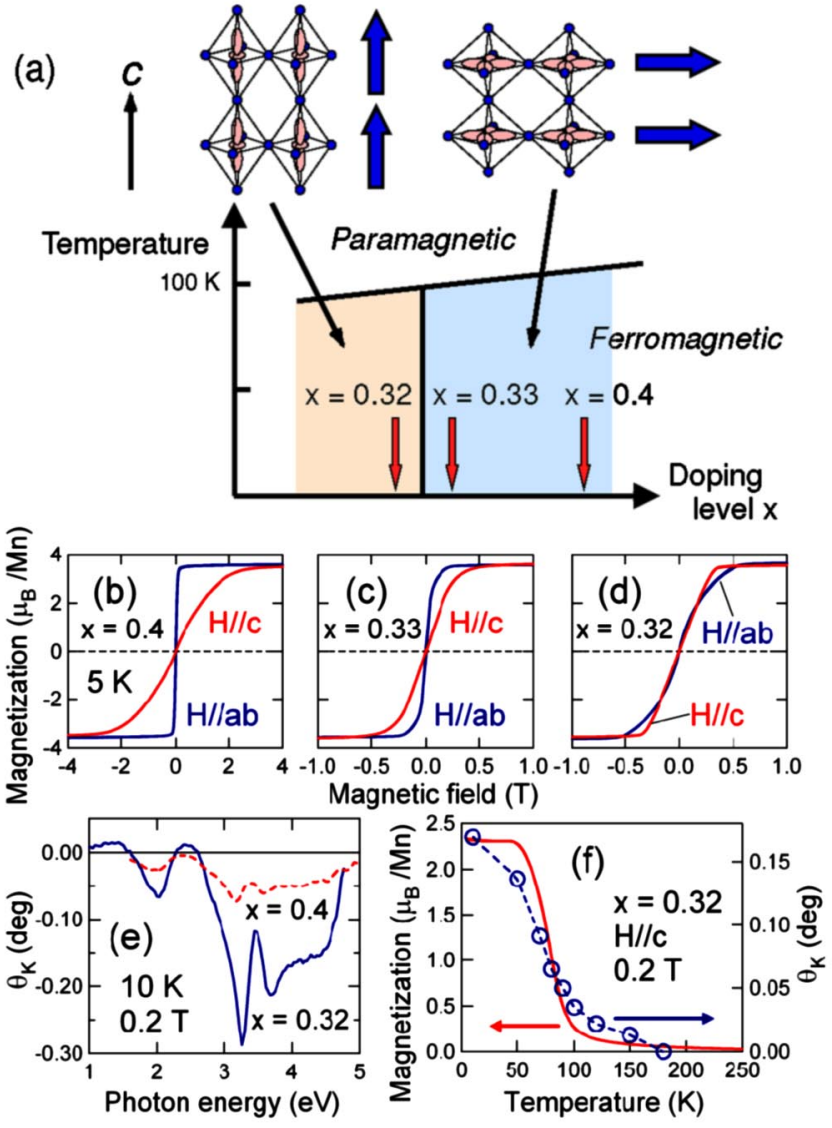

FIG. 1 (color online). Magnetic and magneto-optical properties of $\mathrm{La}_{2-2 x} \mathrm{Sr}_{1+2 x} \mathrm{Mn}_{2} \mathrm{O}_{7}$. (a) Schematic phase diagram. Ferromagnetic spin order (thick arrows) is drawn in the upper part with $\mathrm{MnO}_{6}$ octahedra and the $e_{g}$ electron orbital occupancy (left: $d_{3 z^{2}-r^{2}}$, right: $d_{x^{2}-y^{2}}$ ). (b) -(d) Magnetization curves at $5 \mathrm{~K}$ for $x=0.4,0.33$, and 0.32. (e) Magneto-optical Kerr rotation $\theta_{K}$ spectra at $10 \mathrm{~K}$ in the field of $0.2 \mathrm{~T}$ along the $c$ axis. They are taken with reflection from the $a b$ surfaces of the samples at $x=$ 0.32 (solid) and 0.4 (broken line) by the optical retardation modulation method [21]. (f) Temperature dependence of $\theta_{K}$ at $3.1 \mathrm{eV}$ (circles) and magnetization (solid line) for $x=0.32(H \|$ $c, 0.2 \mathrm{~T}$ ). The broken line in (f) is merely a guide to the eyes.

estimated to be about $180 \mathrm{~nm}$. Although the presence of closure domains near the crystal surface was revealed in the $x=0.32$ compound under no magnetic field [17], the observed large $\theta_{K}$ shown in Figs. 1(e) and 1(f) suggests that their thickness decreases well below the optical penetration depth with the application of the magnetic field ( $\| c$ axis).

Figures 2(a)-2(c) show the time evolution of $\Delta \theta_{K}$ at $x=0.4$ where the magnetization easy axis is parallel to the $a b$ plane. The positive $\Delta \theta_{K}$ represents the decrease of the magnetization (the component along $H \| c$ axis). An intense oscillation due to the precessional motion of the magnetization is observed. But, when the field is increased and the magnetization is saturated, it vanishes away as shown in Fig. 2(c) $(H=2.93 \mathrm{~T})$. In the high field, the rise time becomes longer, reaching about 500 ps. The field
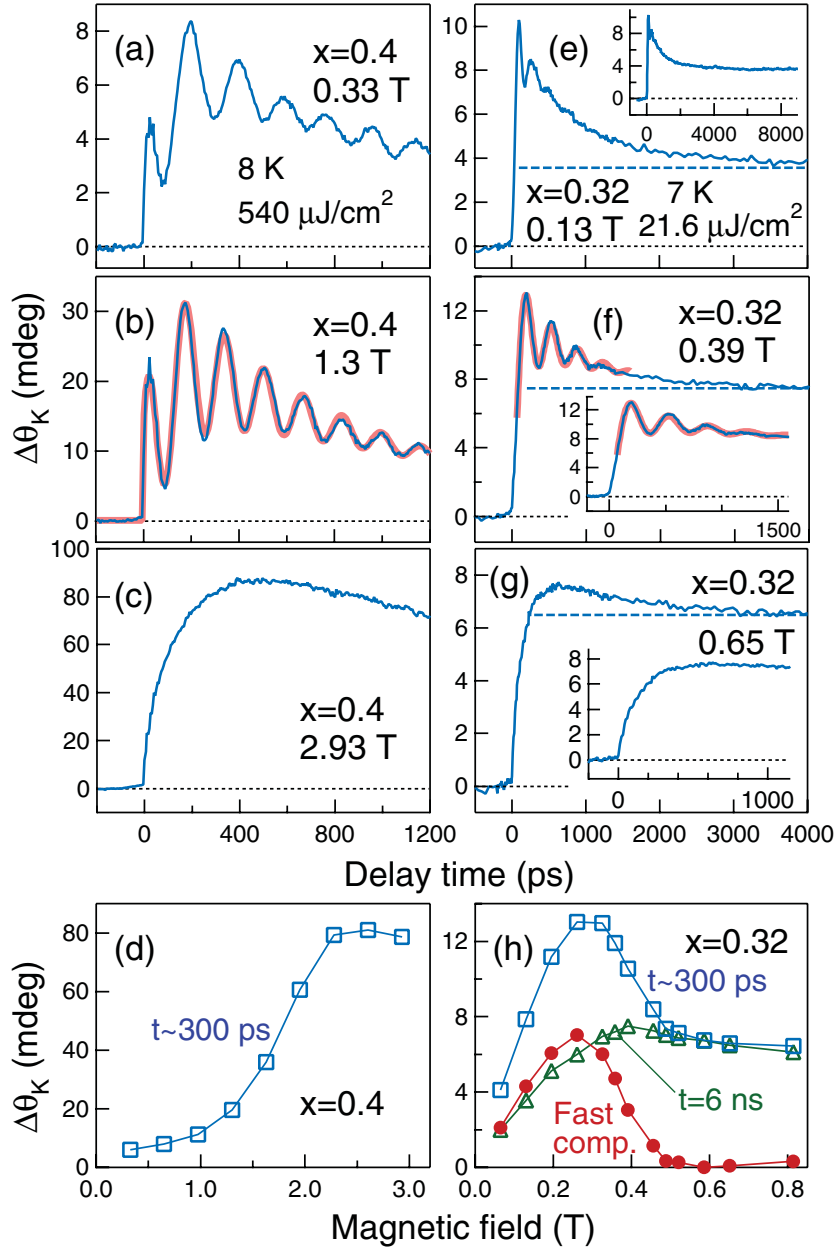

FIG. 2 (color). Time evolution of $\Delta \theta_{K}$ and the field dependence. (a)-(c) $\Delta \theta_{K}(t)$ for $x=0.4$ at temperature $T=8 \mathrm{~K}$. The excitation density is $D_{e}=540 \mu \mathrm{J} / \mathrm{cm}^{2}$ [22]. (d) Field dependence of the $\Delta \theta_{K}$ at $t \sim 300 \mathrm{ps}$ for $x=0.4$. (e) $-(\mathrm{g}) \Delta \theta_{K}(t)$ for $x=0.32$ at $T=7 \mathrm{~K}, D_{e}=21.6 \mu \mathrm{J} / \mathrm{cm}^{2}$. Horizontal broken lines represent the $\Delta \theta_{K}$ at $6 \mathrm{~ns}$ (the magnitude of the slow decay component). The red curves in (b) and (f) are the fitting ones [19]. (h) Field dependence of the $\Delta \theta_{K}$ for $x=0.32$ at $t \sim 300 \mathrm{ps}$ (squares) and $t=6 \mathrm{~ns}$ (triangles). The intensity of the fast component (closed circles) is obtained by $\Delta \theta_{K}(300 \mathrm{ps})-\Delta \theta_{K}(6 \mathrm{~ns})$. The solid lines in (d) and (h) are merely guides to the eyes.

dependence of the magnitude of the nonoscillating background at the delay time $t \sim 300 \mathrm{ps}$ [to avoid the influence of the oscillation, this is obtained by averaging the $\Delta \theta_{K}(t)$ from 100 to $500 \mathrm{ps}$ ] is presented in (d). This field dependence similar to the magnetization curve [Fig. 1(b), $H \| c$ ] is quite normal. The background signal can be unambiguously ascribed to thermal demagnetization from the temperature dependence shown in Fig. 3(a). The $\Delta \theta_{K}(300 \mathrm{ps})$ is sharply enhanced around $110 \mathrm{~K}$, a little lower than $T_{c}$. It is analogous to the temperature derivative of the magnetization, $d M / d T$, shown in Fig. 3(b) (solid line) taken at the same field [18]. This suggests the relation of the thermal demagnetization that $\Delta \theta_{K}(T) \propto \Delta M(T)=M(T+\Delta T)-$ $M(T)$ with the photoheating $\Delta T$ [6]. The slow rise of about 


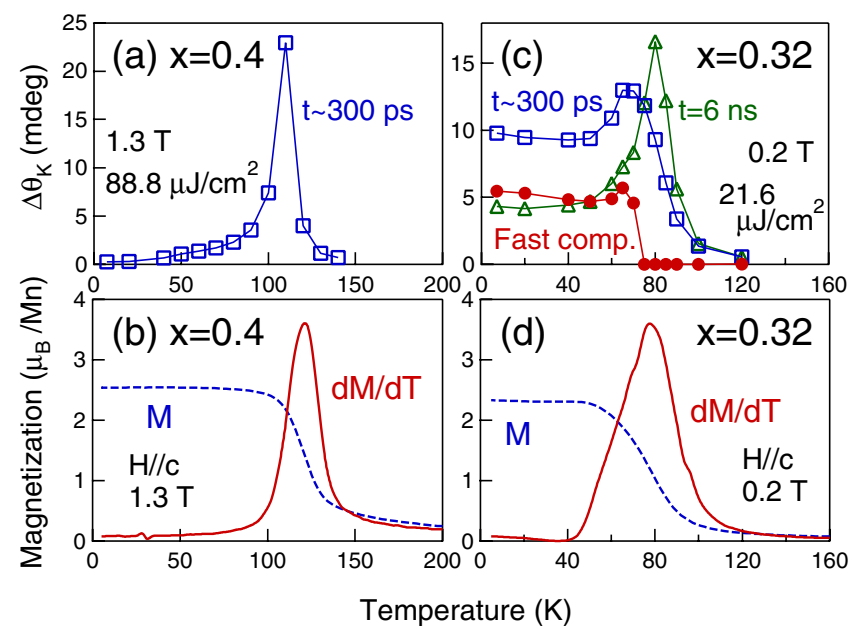

FIG. 3 (color online). Temperature dependence of $\Delta \theta_{K}$ and magnetization. (a) $\Delta \theta_{K}$ at $t \sim 300 \mathrm{ps}$ for $x=0.4(H=1.3 \mathrm{~T}$, $D_{e}=88.8 \mu \mathrm{J} / \mathrm{cm}^{2}$ ). (b) Magnetization (broken) and its first derivative (solid line) for $x=0.4$ in the same field as (a). (c) $\Delta \theta_{K}$ for $x=0.32\left(H=0.2 \mathrm{~T}, D_{e}=21.6 \mu \mathrm{J} / \mathrm{cm}^{2}\right)$ at $t \sim$ $300 \mathrm{ps}$ (squares, obtained by averaging) and $6 \mathrm{~ns}$ (triangles). The fast component (closed circles) is derived by $\Delta \theta_{K}(300 \mathrm{ps})-$ $\Delta \theta_{K}(6 \mathrm{~ns})$ [23]. (d) Magnetization and its first derivative for $x=$ 0.32 in the same field as (c).

500 ps observed at the high field [Fig. 2(c)] gives the time constant of this process, and should be governed by the spin-lattice interaction $[3,6]$.

Figures $2(\mathrm{e})-2(\mathrm{~g})$ show the $\Delta \theta_{K}(t)$ at $x=0.32$ where the magnetization easy axis is parallel to the $c$ axis. In low fields [(e),(f)], the rise is relatively fast, and followed by the clear oscillation due to the magnetization precession. Practically, the rise time (about 200 ps) is limited by the $1 / 2$ of the oscillation period. When the field becomes high enough to saturate the magnetization $[(\mathrm{g})$ at $0.65 \mathrm{~T}]$, the oscillation disappears again, and the rise time becomes longer to be about $500 \mathrm{ps}$ which is comparable to that for $x=0.4$ in the high field. As can be seen in Figs. 2(e) $-2(\mathrm{~g})$, two decay components are observed at $x=0.32$; the fast component has a lifetime of about $1 \mathrm{~ns}$ and the slow one [the amplitudes, $\Delta \theta_{K}(6 \mathrm{~ns})$, are indicated by the broken lines in the figure] has that much longer than $10 \mathrm{~ns}$. The slow component shows enhancement around $80 \mathrm{~K}$ just below $T_{c}$ [triangles in Fig. 3(c), $\Delta \theta_{K}(6 \mathrm{~ns})$ ], and this indicates that this component is mainly coming from the thermal demagnetization.

On the other hand, the fast decay component for $x=0.32$ (the lifetime $\sim 1 \mathrm{~ns}$ ) shows qualitatively different temperature dependence. It can be seen that the $\Delta \theta_{K}(300 \mathrm{ps})$ for $x=0.32$ plotted as squares in Fig. 3(c) is very large even when the temperature is well below $T_{c}$. It is in contrast with that for $x=0.4$ [Fig. 3(a)] in spite of the same delay time $t \sim 300 \mathrm{ps}$. The most important point is that the intensity of the fast decay component [closed circles in Fig. $\left.3(\mathrm{c}), \Delta \theta_{K}(300 \mathrm{ps})-\Delta \theta_{K}(6 \mathrm{~ns})\right]$ is almost flat below $70 \mathrm{~K}$ and shows no enhancement around $T_{c}$. It looks more like following the magnetization $M \| c$ itself [Fig. 3(d), broken line] and disappears above $T_{c} \sim 80 \mathrm{~K}$. This indicates that the origin for the fast component cannot be attributed to thermal processes. One of the candidates of the origin of the fast component might be the magnon excitation during the energy relaxation of photoexcited carriers. But this cannot reasonably explain the suppression of this component under the magnetic field as low as $0.3-$ $0.5 \mathrm{~T}$ [Fig. 2(h), closed circles]. We consider that the fast component is originated from the declining of the magnetization from the $c$ axis to the $a b$ plane induced by the photoinduced directional change of the magnetic anisotropy which will be described below. Then the $c$-axis component of $M$ decreases, and the positive $\Delta \theta_{K}$ (the fast component) appears.

The magnetic anisotropy change is observed through the magnetization precession. From the curve fitting procedure as shown by red lines in Figs. 2(b) and 2(f) [19], we have obtained the precession frequency $\omega=6.08 \mathrm{GHz}$ and the oscillation phase $\phi=0.85 \pi$ for $x=0.4$, and $\omega=$ $2.85 \mathrm{GHz}, \phi=-0.08 \pi$ for $x=0.32$. The phase difference is $\sim \pi$ between the two compounds, thereby suggesting that the respective precessions are in antiphase motion. Such a difference in the initial phase of the oscillation is attributable to the different origin of the anisotropy change. The excitation process of the precession is schematically illustrated in Fig. 4. In the case of $x=0.4$ [Fig. 4(a)], the precession comes from the attenuation of the anisotropy field $H_{a}$ parallel to the surface ( $a b$ plane) $[8,9]$. The $H_{a}$ includes both the contributions from the magnetocrystalline anisotropy and the shape anisotropy associated with the surface (demagnetizing field) [20]. Then, the attenuation of the $H_{a}$ arises from the thermal decrease of the demagnetizing field. In this case, the effective field (the

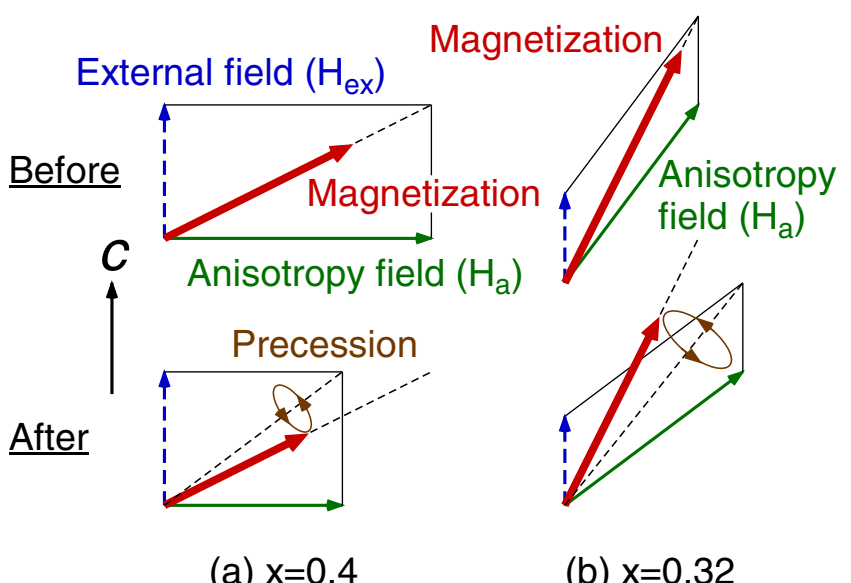

FIG. 4 (color online). Excitation process of magnetization precession for (a) $x=0.4$ and (b) $x=0.32$. Thick arrows are the magnetization. In both the cases, external field (broken arrows) is along the $c$ axis. Anisotropy field (thin arrows) is parallel to the $a b$ plane for $x=0.4$. It should be parallel to the $c$ axis for $x=0.32$ in the bulk, but is assumed to be fractionally tilting due to the demagnetizing field at the sample surface [20]. 
sum of $H_{a}$ and the external field $H_{\mathrm{ex}}$ ) swings slightly toward the $c$ axis. As the magnetization starts to precess around this new field, the $c$-axis component is increased for the first half period after $t=0$. This can be recognized in Figs. 2(a) and 2(b) as the negative change of $\Delta \theta_{K}(t)$ right after $t=0$. In the case of $x=0.32$, on the contrary, the $\Delta \theta_{K}(t)$ shows positive change right after $t=0$ at the initial stage of precession [Fig. 2(f), inset]. This indicates that the directional change of the effective field is quite the opposite of $x=0.4$, i.e., it swings away from the $c$ axis [Fig. 4(b)]. This means that the $H_{a}$, originally tilted from the $c$ axis due to the demagnetizing field [20], further deviates from the $c$ axis at $t=0$ upon photoirradiation, and so does the effective field $\left(H_{a}+H_{\mathrm{ex}}\right)$. Then the precession around this new field starts so as to decrease the $c$-axis component for the first half period. It emerges as the positive change of $\Delta \theta_{K}(t)$ in Fig. 2(f). This can occur at $x=0.32$, which is very close to the phase boundary where the magnetization easy axis changes the direction [Fig. 1(a)], because the photoinduced crossover of the boundary will change the magnetocrystalline anisotropy from the $c$ axis to the $a b$ plane.

We can understand the field dependence of $\Delta \theta_{K}(t)$ at $x=0.32$ shown in Fig. 2(h), attributing the fast decay component to the magnetization declining induced by the above anisotropy change. While the thermal part of $\Delta \theta_{K}$ (at $6 \mathrm{~ns}$, triangles) simply follows the magnetization curve [Fig. 1(d), $H \| c$ ], the fast component [ $\Delta \theta_{K}(300 \mathrm{ps})-\Delta \theta_{K}(6 \mathrm{~ns})$, closed circles in Fig. 2(h)] shows steep decrease from 0.2 to $0.5 \mathrm{~T}$. This is understood as the suppression of the magnetization declining (by the anisotropy change) due to high $H_{\mathrm{ex}}$. The disappearance of the precession in the high field [Fig. 2(g)] is explained in the same way. On the other hand, the disappearance of the precession for $x=0.4$ in the high field [Fig. 2(c)] has a different reason. In this case, when the $H_{\mathrm{ex}}$ is high enough to saturate the magnetization along the $c$ axis (the hard axis), there remains no room for precession which moves the magnetization vector so as to increase the $c$-axis component [Fig. 4(a)].

Last of all, we estimate how large the magnetization declining is. The magnitude of the fast component at $x=0.32$ is linear in excitation density $D_{e}$, and approximately obeys the relation that $\Delta \theta_{K}=D_{e} \times$ $0.62\left[\mathrm{mdeg} / \mu \mathrm{J} \mathrm{cm}^{-2}\right]$ at $H=0.33 \mathrm{~T}$ and $T=7 \mathrm{~K}$ when $D_{e}<5 \mu \mathrm{J} / \mathrm{cm}^{2}$. Taking account of the penetration depth and the reflection loss of the excitation light, we found that the evaluated coefficient corresponds to the declining of about $120 \mathrm{Mn}$ moments per one photon, from the $c$ axis to the $a b$ plane. This amount seems to be reasonable because one photon creates one itinerant photohole, which is equal to raising the doping level $x \sim 0.01$ in the volume of 100 vicinal Mn sites. The increase of $x \sim 0.01$ is enough for the anisotropy change of the $x=0.32$ compound (Fig. 1).

In summary, we have observed the photoinduced directional change of the magnetocrystalline anisotropy in a bilayered perovskite manganite $\mathrm{La}_{2-2 x} \mathrm{Sr}_{1+2 x} \mathrm{Mn}_{2} \mathrm{O}_{7}$ near the boundary of two ferromagnetic phases with different anisotropy by means of the time-resolved measurement of the magneto-optical Kerr effect. At $x=0.32$, the anisotropy field swings away from the $c$ axis upon photoexcitation, which induces the declining of as many as $120 \mathrm{Mn}$ moments per one photon to the $a b$ plane.

We would like to thank M. Konoto for helpful discussions on the micromagnetic state.

*Present address: Bell Laboratories, Lucent Technologies, Murray Hill, NJ 07974, USA.

[1] S. Koshihara et al., Phys. Rev. Lett. 78, 4617 (1997).

[2] K. Matsuda et al., Phys. Rev. B 58, R4203 (1998).

[3] A. I. Lobad et al., Phys. Rev. B 63, 060410(R) (2001).

[4] J. Hohlfeld et al., Phys. Rev. B 65, 012413 (2001).

[5] L. Guidoni et al., Phys. Rev. Lett. 89, 017401 (2002).

[6] T. Ogasawara et al., Phys. Rev. Lett. 94, 087202 (2005).

[7] A. V. Kimel et al., Nature (London) 429, 850 (2004).

[8] M. van Kampen et al., Phys. Rev. Lett. 88, 227201 (2002).

[9] Q. Zhang et al., Phys. Rev. Lett. 89, 177402 (2002).

[10] S. V. Vonsovskii, Magnetism (John Wiley \& Sons, New York, 1974), Vol. 2, pp. 938-960.

[11] J. Kanamori, in Magnetism, edited by G. T. Rado and H. Suhl (Academic Press, New York, 1963), pp. 127-203.

[12] S. Chikazumi, Physics of Magnetism (John Wiley \& Sons, New York, 1964), pp. 128-160.

[13] T. Kimura et al., Phys. Rev. Lett. 81, 5920 (1998).

[14] M. Kubota et al., J. Phys. Soc. Jpn. 69, 1606 (2000).

[15] T. G. Perring et al., Phys. Rev. Lett. 87, 217201 (2001).

[16] T. Ishikawa et al., Phys. Rev. B 62, 12354 (2000).

[17] M. Konoto et al., Phys. Rev. B 71, 184441 (2005).

[18] The small difference of the peak position between Figs. 3(a) and 3(b) is caused by the rather high excitation density, i.e., large $\Delta T$.

[19] The fitting function has a formula of $\Delta \theta_{K}(t)=\Delta \theta_{B}+$ $\Delta \theta_{O}$, where $\Delta \theta_{B}(t)=\left\{1-\exp \left(-t / \tau_{r}\right)\right\}\left\{I_{f} \exp \left(-t / \tau_{f}\right)+\right.$ $\left.I_{S} \exp \left(-t / \tau_{s}\right)\right\}$ denotes the nonoscillating background component. $\Delta \theta_{O}=I_{O} \cos (\omega t+\phi) \exp \left(-t / \tau_{d}\right)$ represents the damped oscillation due to magnetization precession.

[20] The magnetic moments near the surface generally tend to become parallel to the surface ( $\| a b$ plane in the present case) to form closed loops of magnetization flux. Thus, the demagnetizing field should be taken to be parallel to the $a b$ plane.

[21] K. Sato et al., Jpn. J. Appl. Phys. 32, 989 (1993).

[22] This is rather higher than that for $x=0.32$, and was necessary to induce the magnetization change even far below $T_{c}$ at $x=0.4$ where the thermal demagnetization is the only efficient process.

[23] Above $75 \mathrm{~K}, \Delta \theta_{K}(6 \mathrm{~ns})$ becomes larger than $\Delta \theta_{K}(300 \mathrm{ps})$ due to the critical slowing down of rise near $T_{c}$. As the fast component becomes too small to be distinguished at the same time, the values are set to be zero above $75 \mathrm{~K}$. 